\title{
Rebound excitability mediates motor abnormalities in Parkinson's disease
}

\author{
Jeongjin Kim ${ }^{1,2} \mathcal{E}$ Daesoo Kim ${ }^{1, *}$ \\ ${ }^{1}$ Department of Biological Sciences, KAIST, Daejeon 34141, ${ }^{2}$ Center for Neuroscience, KIST, Seoul 02792, Korea
}

\begin{abstract}
Parkinson's disease (PD) is a debilitating disorder resulting from loss of dopamine neurons. In dopamine deficient state, the basal ganglia increases inhibitory synaptic outputs to the thalamus. This increased inhibition by the basal ganglia output is known to reduce firing rate of thalamic neurons that relay motor signals to the motor cortex. This 'rate model' suggests that the reduced excitability of thalamic neurons is the key for inducing motor abnormalities in PD patients. We reveal that in response to inhibition, thalamic neurons generate rebound firing at the end of inhibition. This rebound firing increases motor cortical activity and induces muscular responses that triggers Parkinsonian motor dysfunction. Genetic and optogenetic intervention of the rebound firing prevent motor dysfunction in a mouse model of PD. Our results suggest that inhibitory synaptic mechanism mediates motor dysfunction by generating rebound excitability in the thalamocortical pathway. [BMB Reports: Perspective 2018; 51(1): 3-4]
\end{abstract}

The brain generates motor signals to organize a spectrum of behavior, ranging from simple to complex. In 1950s, Eccles and colleagues established a simple motor circuit model in the spinal cord: principal motor neurons transmit motor signals that trigger muscle contractions by releasing the excitatory neurotransmitter, acetylcholine, whereas local interneurons inhibit this excitatory mechanism by releasing $\gamma$-aminobutyric acids (GABA). In higher motor circuits in the brain, some GABA-producing neurons play predominant roles in the

*Corresponding author. E-mail: daesoo@kaist.ac.kr

https://doi.org/10.5483/BMBRep.2018.51.1.004

Received 29 December 2017

Keywords: Basal ganglia, Parkinson's disease, Rebound firing, Thalamus, T-type $\mathrm{Ca}^{2+}$ channels

Abbreviations: VL, ventrolateral thalamic nuclei; GPm, GLobus pallidus medial nuclei

Perspective to: Jeongjin Kim, et al. Inhibitory Basal Ganglia Inputs Induce Excitatory Motor Signals in the Thalamus. Neuron, volume 95:5, 1181-1196. http://dx.doi.org/10.1016/j.neuron.2017.08.028 control of motor behavior, suggesting that relevant motor information can also be relayed via inhibitory synaptic mechanisms.

The basal ganglia - that play a critical role in action selection, patterning and coordination - consist predominantly $(96 \%)$ of inhibitory neurons and produce mainly inhibitory outputs. The activity of these inhibitory neurons is modulated by dopamine; in dopamine-deficient states, their inhibitory input to target areas is increased and may contribute to motor dysfunction in Parkinson's disease (PD) and L-DOPA-responsive dystonia. Given that patients with dopamine deficiency show multiple motor abnormalities - such as akinesia, rigidity, tremor, and gait abnormalities - the inhibitory input provides by the basal ganglia conveys diverse motor information.

Pioneering studies have suggested how enhanced inhibitory synaptic transmission from the basal ganglia exerts pathological effects. According to the rate model, inhibitory input decreases overall firing rate in target neurons, thereby interfering with their motor functions (Albin et al., 1989). In a second model, inhibited neurons generate low-threshold burst firing, that depend on the activation of T-type $\mathrm{Ca}^{2+}$ channels and exert a strong post-synaptic impact. A third model posits that rhythmic inhibition induces oscillations, that synchronizes target neurons at specific frequencies and may result in abnormal processing of motor information. However, because PD patients show phenomena consistent with all three of these hypotheses, it has been challenging to uncover the key mechanism involved. The lack of a robust mouse model that precisely mimics the physiological and behavioral consequences of dopamine deficiency has limited analysis of the circuitbased functions of diverse basal ganglia outputs.

Here we used optogenetic approach to control the activity of specific inhibitory inputs (GPm, globus pallidus medial) to the ventro lateral thalamus $(\mathrm{VL})$ and examined the effects of such manipulation in wild-type (WT) mice and in a mouse model of dopamine deficiency. Through this approach, we found that in the VL thalamus, T-type $\mathrm{Ca}^{2+}$ currents $\left(I_{T}\right)$ were involved in neither the generation of low-threshold burst firing nor inhibition-induced oscillations. We instead propose that T-type $\mathrm{Ca}^{2+}$ channels induce rebound firing that induces PD-like motor abnormalities, such as akinesia, rigidity, and tremor, the severity of which is proportional to the number of neurons with rebound firing. In CaV3.1-KO mice lacking $I_{T}$, rebound

ISSN: 1976-670X (electronic edition)

Copyright (c) 2018 by the The Korean Society for Biochemistry and Molecular Biology

(c) This is an open-access article distributed under the terms of the Creative Commons Attribution Non-Commercial License (http://creativecommons.org/licenses/by-nc/4.0) which permits unrestricted non-commercial use, distribution, and reproduction in any medium, provided the original work is properly cited. 


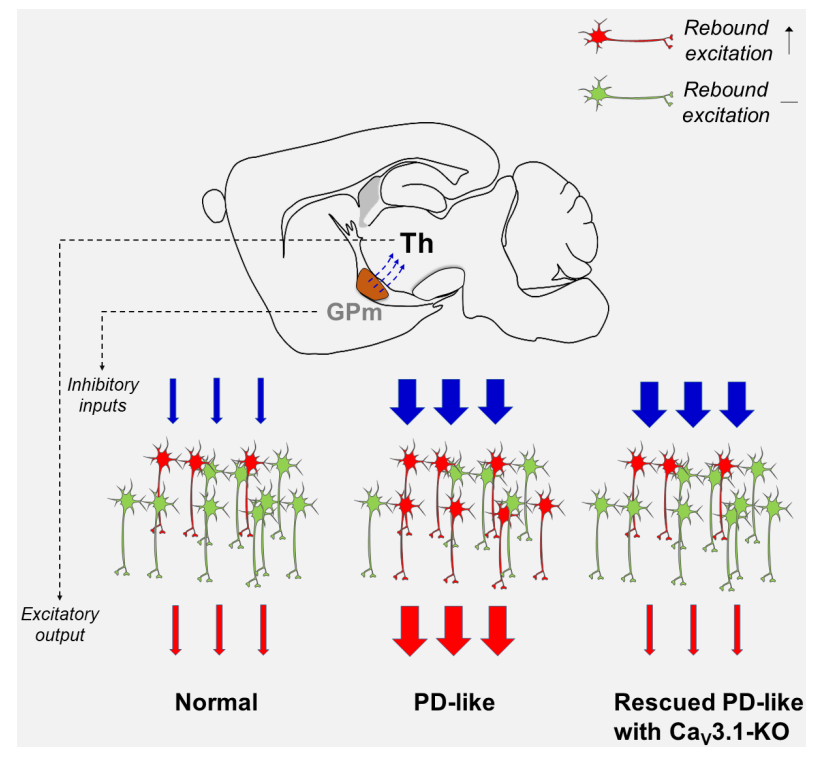

Diagram 1. Excitatory motor signals in the thalamus generate multiple PD-like motor symptoms. In low dopamine condition, enhanced basal ganglia inputs to the thalamus generate rebound firing (rebound excitation) in many thalamic neurons. Suppression of rebound firing by CaV3.1 $\mathrm{KO}$ or direct inhibition of thalamic activity rescue PD-like motor problems.

firing is reduced and fails to induce motor responses (Diagram 1). In a mouse model of PD, reduction of rebound excitability of thalamic neurons by genetic and optogenetic means abolishes motor abnormalities.

What is the physiological role of rebound firing of $V L$ neurons? Considering that the $\mathrm{VL}$ area has a somatotopic relationship with the cortex, the production of rebound firing in many VL neurons may, in turn, increase muscle tone in many body parts simultaneously. Maintenance of muscle tension against gravity is critical during resting postures. It is interesting to note that PD patients also show non-specific recruitment of muscles that may lead to whole-body rigidity and tremors, especially during the resting state. In conjunction with our results indicating a postulated role for coincident rebound firing in body stabilization, this may indicate that abnormal potentiation of rebound firing may produce PD condition by causing abnormal recruitment of muscles.

Finally, our study sheds light on how to cure PD. First, reducing the inhibitory synaptic inputs to the thalamus could reduce rebound excitation. Second, direct reduction of rebound firing of VL neurons by blockade of CaV3.1 may also relieve motor symptoms. Revealing the precise molecular mechanisms underlying the inhibition-induced rebound excitability may provide clues to novel therapeutic targets, particularly for neurological disorders associated with enhanced inhibition.

\section{ACKNOWLEDGEMENTS}

This study was supported by grants from the National Leading Research Laboratory Program (2016-015167) and an intramural funding from the Korea Institute of Science and Technology (2E27372). 